AIAA Paper 2003-6327, Space 2003 Conference \& Exposition

\title{
ENGINEERING LESSONS LEARNED AND TECHNICAL STANDARDS INTEGRATION: CAPTURING KEY TECHNOLOGIES FOR FUTURE SPACE MISSIONS
}

\author{
By *Daniel P. Mellen, *Danny Garcia, and **William W. Vaughan \\ (*NASA Marshall Space Flight Center, ${ }^{* *}$ University of Alabama in Huntsville)
}

\begin{abstract}
$\underline{\text { Abstract }}$
Capturing engineering lessons learned derived from past experiences and new technologies, then integrating them with technical standards, provides a viable process for enhancing engineering capabilities. The development of future space missions will require ready access, not only to the latest technical standards, but also to lessons learned derived from past experiences and new technologies. The integration of this information such that it is readily accessible by engineering and programmatic personnel is a key aspect of enabling technology. This paper addresses the development of a new and innovative Lessons Learned/Best Practices/Applications Notes--Standards Integration System, including experiences with its initial implementation as a pilot effort within the NASA Technical Standards Program. Included are metrics on the Program, feedbacks from users, future plans, and key issues that are being addressed to expand the System's utility. The objective is the enhancement of engineering capabilities on all aspects of systems development applicable to the success of future space missions.
\end{abstract}

\section{Introduction/Background}

Government and commercial aerospace organizations in the United States who are either planning or engaged in efforts related to the development of new or improved manned and un-manned space vehicles are benefiting from the use of technical standards and lessons learned gained from previous experiences. Technical standards are an essential aspect of all engineering development efforts, but are of particular significance in the aerospace industry. In 1997 NASA established an Agencywide Technical Standards Program under the direction of the NASA Chief Engineer.

This Material is declared a work of the U.S. Government and is not subject to copyright protection in the United States.
The Program, via its Web site http://standards.nasa.gov, has undertaken an extensive effort to make available technical standards, including relating them to lessons learned data sources, for use in the development of the Agency's programs and related engineering activities.

NASA is currently involved in the development of an Orbital Space Plane (OSP), a next generation space vehicle designed to provide a crew rescue and crew transport capability to and from the International Space Station. This effort will benefit from the lessons learned from previous vehicle designs, component developments, and operations. The success of this critical development effort is largely dependent on the applicable lessons learned that are identified and applied, as well as the application of relevant technical standards. Properly applied technical, standards and lessons learned enhance engineering capabilities. The application of standards and lessons learned will reduce the cost of the engineering design and increase system reliability.

The roots of all technical standards are buried in lessons learned, and the roots of lessons learned extend downward into the depths of our experiences. These experiences, both positive and negative, form the basis of lessons learned data sources. To be significant, lessons learned must have a real impact on the program's function or procedure. Lessons learned must be screened and validated to assure that they contain accurate technical content. In order for the lessons to be effective, they must also be applicable and address a specific design process or decision that mitigates risk, increases safety and reliability, or leads to an improved design or process. Once captured, lessons can then be integrated with technical standards to form a powerful engineering information source to enhance not only space vehicle development efforts, but to improve organizational engineering capabilities. This paper is based on the experiences gained from the implementation of the NASA Technical Standards Program Web site, and a pilot study on the integration of lessons learned/best practices/application notes with technical standards. 


\section{Discussion}

The search for relevant lessons learned information can be challenging because most lessons learned are not written in a standardized format and are disseminated in various mediums. They are documented in technical papers and memos, professional journals, and databases, and can be found in numerous forms. Locating lessons relating to particular areas of technical expertise or technical disciplines is equally as challenging. To overcome this obstacle, the simple notion of integrating lessons learned with technical standards has proven to be a very viable means by which the engineering community can locate and infuse these lessons into the design and development of specific programs and projects.

Examples of lessons learned data sources incorporated into the NASA Technical Standards Program Web site include:

- NASA/Headquarters- Lessons Learned Information System.

- NASA/Glenn Research Center- Frequently Asked Questions on Failures.

- NASA/Kennedy Space Center- Cryogenic Transfer Mechanical Design.

- NASA/Goddard Space Flight Center- Systems Engineering Lessons Learned.
- AIAA Satellite Mission Operations Best Practices.

- NASA/Langley Research Center- Lessons for Software Systems.

With the lessons learned - standards integration effort comes the problem of locating lessons focused on a specific technical category. To ease this problem, the NASA Technical Standards Program developed the technical categorization taxonomy (Figure 1.). These categories are based on those developed by the Department of Defense and are the basis for both the NASA technical standards and the lessons learned categorization. In addition to enhancing the search capability by the end user of the System, this classification enables the user to more readily locate both standards and lessons learned applicable to the discipline of interest.

During the life cycle of a program, the use of lessons learned and standards occur at the various appropriate phases to maximize the use of technology (Figure 2.). This technology is then reviewed by the managers and technical experts to determine the best direction of the program. As a result of technical decisions and accomplishments at the various phases of the life cycle, new lessons learned can then be identified and documented and related to technical standards for use on future program developments. Thus, the experience and knowledge gained can be readily made available to all concerned.

\begin{tabular}{|c|c|}
\hline Discipline Category & Title \\
\hline 0000 & Documentation and Configuration Management, Program Management \\
\hline 1000 & Systems Engineering and Integration, Aerospace Environments, Celestial Mechanics \\
\hline 2000 & Computer Systems, Software, Information Systems \\
\hline 3000 & Human Factors and Health \\
\hline 4000 & Electrical Systems, Electronlcs, Avionics/Control Systems, Optics \\
\hline 5000 & Structures/Mechanical Systems, Fluid Dynamics, Thermal, Propulsion, Aerodynamics \\
\hline 6000 & Materials and Processes, Parts \\
\hline 7000 & System Test, Analysis, Modeling, Evaluation \\
\hline 8000 & Safety, Quality, Rellability, Maintainability \\
\hline 9000 & Operations, Command, Control, Telemetry/Data Systems, Communications \\
\hline
\end{tabular}

Figure 1. Technical Categorization Taxonomy 


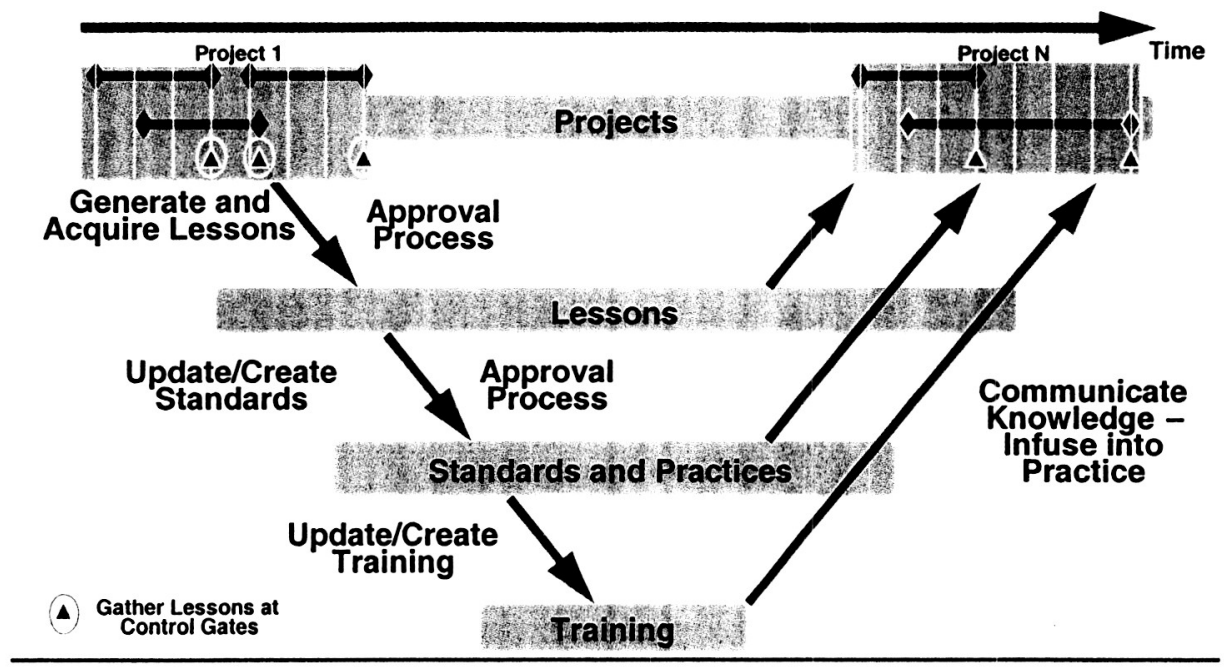

Figure 2. Infusion into Programs and Projects

\section{Importance and Explanation of Linking of Lessons Learned with Standards}

At a glance it may appear that the linking of lessons learned to standards is an easily achievable task. After reviewing the data and resources during the pilot effort, it became clear that a major amount of time and effort is required to ensure a qualified product. The initial effort requires the location of lessons learned, whether electronic or paper form. Once this is accomplished, a detailed evaluation of the lesson learned must be performed to determine the validity of the lesson in relation to aerospace engineering applications. This is an important aspect of the undertaking relative to success of the endeavor. After reading the lesson, a search of related technical standards must be accomplished. This entails a detailed screening of technical standards that must be performed using specific keywords based on the content retrieved by the evaluation of the lesson. This also requires a thorough technical review. The use of advanced search engine capabilities will enable the reviewer to achieve better results in this search and in a more efficient manner. After reviewing the summary of the lessons learned evaluation results, the reviewer must then physically read the standard relative to the lesson learned evaluation to ensure the validity of the match. An independent reviewer should then verify the results. After completion of this task, the applicable lessons learned are linked with the standards in the database, ready for the next user of the System.
Lessons Learned/Best Practices/Applications Notes-Standards Integration System

The NASA's Lesson Learned/Best Practice/ Application Notes-Technical Standards Integration System has its origins in the initial technical standards program requirements that were established by the NASA Chief Engineer. The purpose of this System is to make available to those users within the <nasa.gov> domain the on-line technical standards linked with lessons learned. The objective is to provide ready access to the most recent technological advances within the aerospace industry.

The Document Summary Page on the NASA Technical Standards Program Web site contains the pertinent information about the particular technical standards document (Figure 3.). The user is provided the title, current revision, status, and an abbreviated scope of the standards document. If there is an application note for the particular document, it is also included. An application note is a brief statement submitted by a user that briefly clarifies or limits the scope, use, or context of a given technical standard. Also contained on this Document Summary Page are lessons learned that have been screened and linked to the particular standard where relevance between the lesson and standard has been determined. 


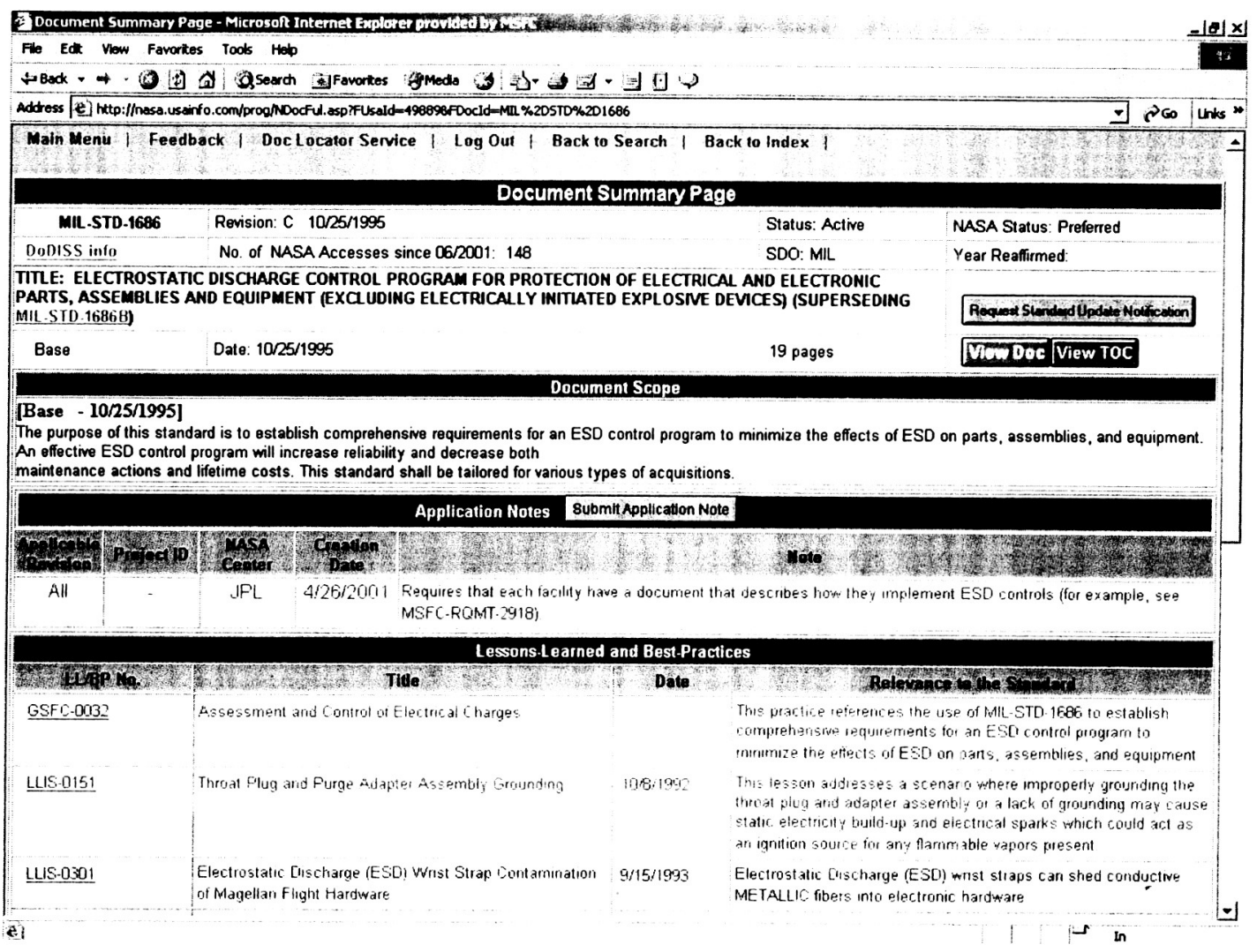

Figure 3. Document Summary Page Example

\section{Example of a Lessons Learned Influencing the Revision of a Standard}

As part of the flight certification of the High Energy Spectroscopic Imager (HESSI) spacecraft, a series of vibration tests at the NASA Jet Propulsion Laboratory (JPL) were conducted. The structural qualification test, a sine-burst test on a shaker table, subjected the spacecraft to a major overtest condition that resulted in significant structural damage to the spacecraft.

The root cause of the overtest condition was the mechanical binding ("stiction" or static friction) between the slip table and the granite mass. It resulted from physical contact between a portion of the slip table and the granite mass caused by a mechanical failure in the shaker's support structure. The stiction caused the shaker system to present highly non-linear gain characteristics to the control system making it impossible for the controller to calculate an appropriate forcing function.
Other contributing factors identified were the lack of facility validation testing, age of the equipment, and test personnel lacking adequate knowledge of the test facility and it's systems. Even though there are inherent risks associated with vibration testing, if these factors had been adequately addressed, then the failure may not have occurred. As a result of this test and the investigation that followed, plus similar vibration tests performed for flight certification of flight hardware, NASA-STD-7004, "Force Limited Vibration Testing" was revised based on the "lessons learned"

\section{Metrics and Feedback}

Metrics play a vital role in providing a true picture of the usage of the information on the NASA Technical Standards Program Web site. NASA tracks a variety of usage metrics for both technical standards and lessons learned. The standards and lessons learned major metrics tracked are the number of accesses and the Top 20 downloads. These are used to gauge the effectiveness of the Web site and also to identify where trends occur. This enables actions to be taken to 
modify the Program content, etc. An example of the Top 20 Lessons Learned data sources accessed since July 2000 is shown in Figure 4.

Feedback from the user is a very important feature of the NASA Technical Standards Program Web site. Not only does it enable improvements to be made on content of the Web site, but it also provides information on its operation. Change requests, complements, observations and questions, and suggestions and comments are routinely entered into the feedback system for a response to the user within 24 hours. This service helps ensure that all users remain comfortable with the performance of the Web site and get the information required to perform their tasks. Since May 2001, 66 change requests, 49 complements, 140 observations, 904 questions, 271 suggestions and comments were received. Incorporation of suggestions and comments has significantly enhanced the Program's content and utility. Essentially all of the questions occurred in the early implementation stages of the Web site and were associated with the institution of a new "culture" for standards access and use. One of the more recent feedbacks was "Regarding your questionnaire on the usage of the NASA Technical Standards Program, I have wanted to state for some time that the program has been an invaluable tool to me in all phases of my job which include almost all categories in the questionnaire. The NASA Technical Standards Program has greatly facilitated my work by providing easy access to applicable standards, specifications, and reports. I have recommended the Program to several colleagues who have also benefited."

A pilot effort was undertaken in June 2003 to help identify the primary usage being made of standards accessed by users within the <nasa.gov> domain. This was done by requesting inputs from those logging on the NASA Technical Standards Program Web site to access full-text standards (Figure 5.). This revealed that the primary uses for the standards products, within the <nasa.gov> domain are in-house research and development activities and development of requirements for programs/project development. This pilot study is still underway and will be expanded, thus providing not only the NASA Technical Standards Program, but managers of the Agency's programs and projects, senior Center and Headquarters managers, etc. with a better appreciation of the importance and use being made of technical standards products.

\begin{tabular}{|l|c|}
\hline \multicolumn{1}{|c|}{ Data Source } & Accesses \\
\hline A History of Aerospace Problems, Their Solutions, Their Lessons & 406 \\
\hline System Engineering "Toolbox" for Design-Oriented Engineers & 120 \\
\hline NASA Lessons Leamed Information System & 119 \\
\hline NASA Preferred Reliability Practices for Design and Test & 115 \\
\hline Flight Project Lessons Leamed Database & 97 \\
\hline Space Engineering Lessons Learned & 90 \\
\hline Systems Engineering Office Lessons Learned & 88 \\
\hline Skylab Lessons Leamed & 88 \\
\hline Software Engineering, Doing Requirements Right the First Time! & 80 \\
\hline Technical Design Review Practices & 70 \\
\hline Fastener Torque and Clamp Force Lessons Learned & 67 \\
\hline Launch Vehicle Design Process: Characterization, Technical Integration, and Lessons Learned & 65 \\
\hline Interplanetary Mission Design Handbook & 56 \\
\hline Working on the Boundaries: Philosophies and Practices of the Design Process & 55 \\
\hline Best Manufacturing Practices & 54 \\
\hline "Non-Magnetic" Materials May Become Magnetized & 53 \\
\hline COTS-Based Software Development: Processes and Open Issues & 51 \\
\hline The Apollo 13 Accident & 46 \\
\hline NASA Electronic Parts and Packaging & 44 \\
\hline Design Guidelines for Assessing and Controlling Spacecraft Charging Effects Spacecraft Charging & 42 \\
\hline Effects & 4 \\
\hline
\end{tabular}

Figure 4. Top 20 Lessons Learned/Best Practices Accessed Via The NASA Technical Standards Program Web site 


\begin{tabular}{|c|c|c|c|c|c|c|}
\hline $\begin{array}{c}\text { Development of } \\
\text { Requirements } \\
\text { for } \\
\text { Program/Project } \\
\text { Development }\end{array}$ & $\begin{array}{c}\text { In- House } \\
\text { Research } \\
\text { and } \\
\text { Development } \\
\text { Activities } \\
\text { (Including } \\
\text { Design, } \\
\text { Analysis, } \\
\text { Testing, Etc.) }\end{array}$ & $\begin{array}{c}\text { Verification of a } \\
\text { Contractor's } \\
\text { Design } \\
\text { and Development } \\
\text { Processes on } \\
\text { Programs/Projects }\end{array}$ & $\begin{array}{c}\text { Acquisition } \\
\text { of Parts or } \\
\text { Materials }\end{array}$ & $\begin{array}{c}\text { Evaluation } \\
\text { of } \\
\text { Proposal(s) }\end{array}$ & $\begin{array}{c}\text { Education } \\
\text { and } \\
\text { Training }\end{array}$ & $\begin{array}{c}\text { Other } \\
\text { Uses }\end{array}$ \\
\hline $23.3 \%$ & $29 \%$ & $18.6 \%$ & $11.5 \%$ & $3.7 \%$ & $8.8 \%$ & $5.1 \%$ \\
\hline
\end{tabular}

Figure 5. Standards Use Questionnaire Results (June-August 2003)

\section{Some Key Issues-An Overview}

\section{Lack of Common Format}

One of the key issues identified regarding the lessons learned data sources is the lack of a common format. A majority of specific lessons learned that have been linked to standards were obtained from the NASA Lessons Learned Information System htp://llis.nisil.gov/. These lessons learned are presented in a common format that contains title, description and lesson learned information sections. Lessons found in other data sources can be in the form of program reports, failure analyses, technical memorandums, technical journal articles, or just a listing of programmatic or technical lessons learned. Therefore, the user must be flexible when searching and reviewing lessons learned data sources linked to standards, or otherwise accessed.

\section{Validation of Lessons Learned}

Before being linked to a technical standard, the lesson must be validated. This takes the effort of dedicated technical engineers to evaluate the lesson in detail and determine if it in fact is worthy to be used as a validated lesson learned. Since the technical community is so diverse, interpretation of the lesson can take many paths. It is critical that much attention be given to engaging technical reviewers having the right talents and engineering experiences. For the best evaluation, a technical working group reviewing the lessons is the preferred method to achieve the most viable final results. After the review is completed, the lesson can be integrated into the System and linked to a relevant technical standard.
Accessibility

In order to make the most of the integration of lessons learned with technical standards and infuse them into the programs and projects, it is preferred that they be identified as part of one or more phases of the program life cycle. Some, if not most, standards required should be determined at the initial program requirements review. Then at subsequent design and development phases, other standards should be identified for use, with control gates installed within the planning to ensure that the lessons and technical standards are addressed and used to ensure a successful mission or program. This will involve a change of culture in the technical community, but it will be one of high value to the program.

\section{Acquisition of Lesson Learned}

Accessing lessons learned is another obstacle that must be overcome. Some lessons may be found electronically over the Internet, and are readily accessible. Others may be in paper form stored in filing cabinets, located on password protected Web sites, or proprietary. In these cases, additional steps are required if they are to be accessible to the user.

\section{Awareness of Data Sources and Content}

To remain current with the available technology, as new lessons are developed and made available, the user must be informed. It is therefore imperative that announcements, such as "What's New" on the NASA Technical Standards Program Web site or emails, be sent to users who register for notification requests within their discipline category. The user must be made aware of the addition of current lessons learned in a timely and user-friendly manner in order to be able to take advantage of current technology. Development of this initiative is currently in the planning stages as part of the pilot program. 


\section{Future}

The importance of integrating of lessons learned with technical standards cannot be stressed too much. The ultimate success of future aerospace programs and projects will depend on the technological advances that are reflected in lessons learned and technical standards. The utilization of this information is a key factor in enhancing engineering capabilities within the aerospace industry. Success becomes achievable as the culture in the aerospace technical community changes to more timely accept the advances and experiences documented in the lessons learned. Integrating lessons learned with technical standards is heavily reliant on current technology and the resulting user interface must being both extensive and user friendly. This will present a unique challenge with today's electronic technology. The implementation of such a system will most assuredly ensure the success of many advanced space mission development efforts.
Bibliography

1. Gorman, Maryann, "The NASA Technical Standards Program: An Interview With The Manager". ASTM Standardization News, July 2003

2. Gill, Paul S., William W. Vaughan, and Danny Garcia, "Lessons Learned and Technical Standards: A Logical Marriage”. ASTM Standardization News, November 2001

3. Gill, Paul S. and William W. Vaughan, "The Changing Climate for Standards". ASTM Standardization News, May 1999

(Prepared: August 28, 2003)

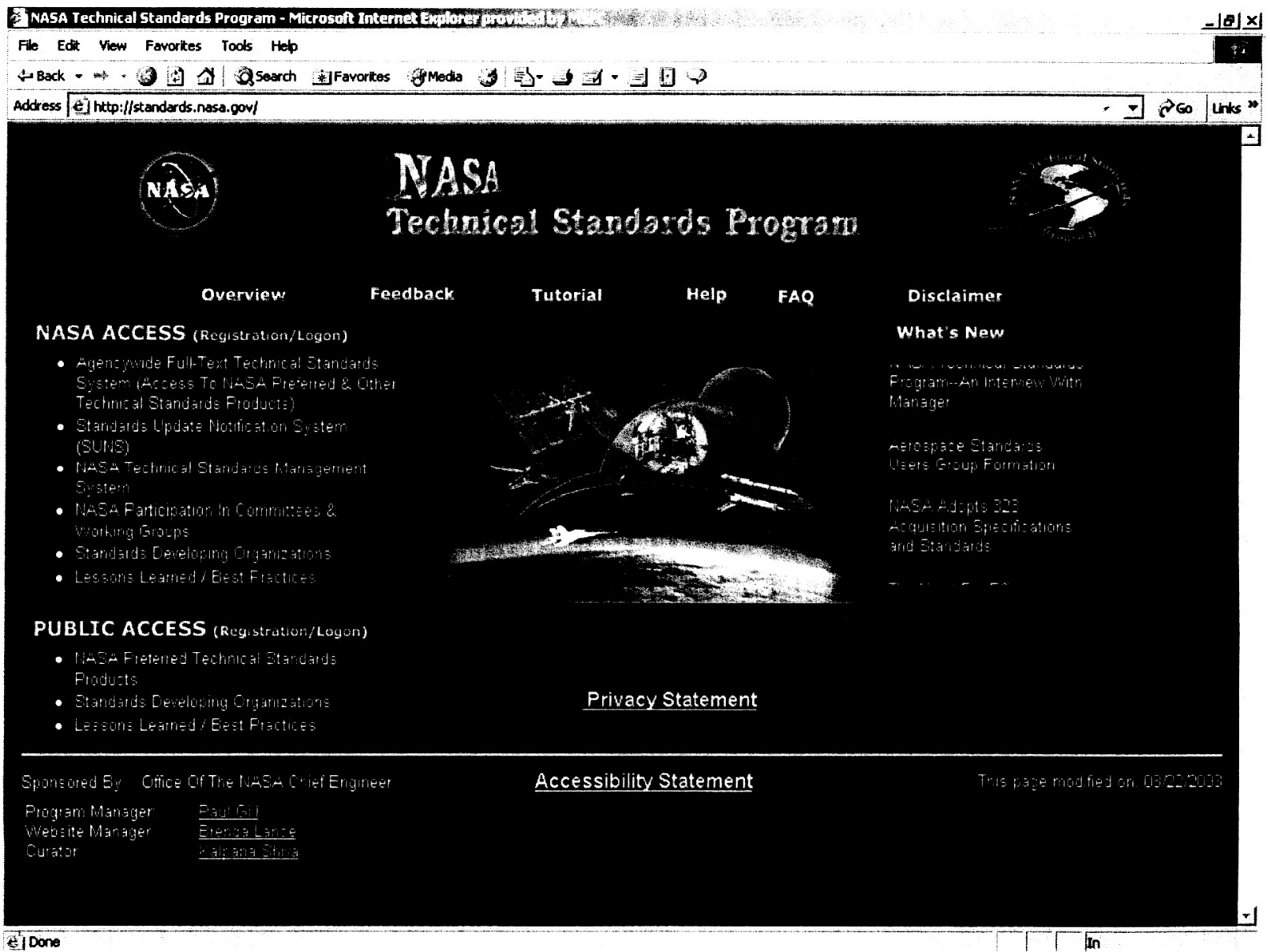

The NASA Technical Standards Program Web site Home Page 